Note: This is a preprint of a paper being submitted for publication. Contents of this paper should not be quoted nor referred to without permission of the author(s).

Submitted to the Materials Research Society 1994 fall meeting, Symposium A, Beam-Solid Interactions for Materials Synthesis and Characterization, ed. by D. E. Luzzi, M. Iwaki, T. F. Heinz, and D. C. Jacobson.

\title{
Linear and Nonlinear Optical Properties of Metal Nanocluster-Silica Composites Formed by Sequential Implantation of $\mathbf{A g}$ and $\mathbf{C u}$
}

\author{
R. A. Zuhr \\ Oak Ridge National Laboratory \\ Oak Ridge, TN
}

R. H. Magruder III, T. A. Anderson, and D. O. Osborne Jr. Vanderbilt University Nashville, TN

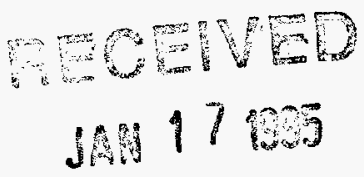

OSTI

\begin{abstract}
Whe submitted manuscript has been authored by contractor of the U.S. Government under contract No. DEAC05-840R21400. Accordingly, the U.S. Government retains a nonexclusive, royalty-free license to publish or reproduce the published form of this contribution, or allow others to do so, for U.S. Government purposes."
\end{abstract}

Prepared by the

Oak Ridge National Laboratory

Oak Ridge, Tennessee 37831

managed by

MARTIN MARIETTA ENERGY SYSTEMS, INC.

for the

U.S. DEPARTMENT OF ENERGY

under contract DE-AC05-84OR21400 


\title{
LINEAR AND NONLINEAR OPTICAL PROPERTIES OF METAL NANOCLUSTER-SILICA COMPOSITES FORMED BY SEQUENTIAL IMPLANTATION OF AG AND CU
}

\author{
R.A. ZUHR*, R.H. MAGRUDER,III**, T.A. ANDERSON**, AND D.O. OSBORNE. JR** \\ * Solid State Division, Oak Ridge National Laboratory, Oak Ridge, TN 37831 \\ **Vanderbilt University, Nashville, TN 37235
}

\begin{abstract}
Nanometer dimension metal colloids were formed in silica by sequential implantation of $\mathrm{Ag}$ and $\mathrm{Cu}$ ions. The $\mathrm{Ag}$ and $\mathrm{Cu}$ were implanted with relative ratios of $\mathrm{Ag}$ to $\mathrm{Cu}$ of 9:3,6:6, and 3:9. The total nominal dose was $12 \times 10^{16} \mathrm{ions} / \mathrm{cm}^{2}$. TEM techniques were used to examine colloid size and size distributions. The linear optical response was measured from 200 to $900 \mathrm{~nm}$. The nonlinear optical properties were measured using the $\mathrm{z}$-scan technique at a wavelength of $570 \mathrm{~nm}$. The linear and nonlinear optical properties were found to be dependent upon the relative ratio of sequentially implanted $\mathrm{Ag}$ to $\mathrm{Cu}$. The results are consistent with effective medium theory.
\end{abstract}

\section{INTRODUCTION}

Sequential ion implantation offers a unique method of forming nanometer dimension metal particles in glass. 1,2,3 It has been demonstrated to significantly alter the composition of the metal particles formed, and hence the electronic structure of the colloids, by forming nanometer dimension "alloy" colloids. As both linear and nonlinear optical properties are a function of the electronic structure of the colloids, sequential implantation can significantiy affect the optical response of these composites. $4,5,6$ The ability to form these "alloy" metal-glass composites could significantly improve the feasibility of these materials as optical devices by enhancing the nonlinear response and by changing the character of that response. ${ }^{7}$ In this paper we report the effects of the relative ratio of sequentially implanted $\mathrm{Ag}$ to $\mathrm{Cu}$ on the formation of the nanosize particles and on the optical response of the composites formed.

\section{EXPERIMENTAL}

$\mathrm{Ag}$ and $\mathrm{Cu}$ ions were sequentially implanted in Corning 7940 high purity silica substrates. The implantation energies were chosen from calculations using TRIM $89^{8}$ to target energies that would overlay the depths of implantation for each metal. The Ag ions were implanted first at $305 \mathrm{keV}$ with substrate temperatures of $\sim 270^{\circ} \mathrm{K}$ and a current density of $\sim 1.3 \mu \mathrm{mmps} / \mathrm{cm}^{2}$. The $\mathrm{Cu}$ ions were implanted sequentially at $160 \mathrm{keV}$ with substrate temperatures of $\sim 270^{\circ} \mathrm{K}$ and a current density of $\sim 3 \mu \mathrm{amps} / \mathrm{cm}^{2}$. The doses used were in ratios, $\mathrm{Ag}$ to $\mathrm{Cu}$, of 9:3, 6:6, and 3:9. Nominal total doses as determined by current integration for the three samples were $12 \times 10^{16}(\mathrm{Ag}+$ $\mathrm{Cu}$ ) ions $/ \mathrm{cm}^{2}$. Rutherford backscattering (RBS) measurements with $2.3 \mathrm{MeV} \mathrm{He}++$ ions were used to measure the depth profiles of implanted species. Planar view samples for transmission electron microscopy (TEM) were prepared by conventional techniques reported elsewhere.

Linear optical absorption measurements were made at room temperature in air from 900 to 200 $\mathrm{nm}$ using a dual beam spectrometer (Cary 5). All samples were measured using an unimplanted sample in the reference beam. The absorption spectra were measured at three different positions on each sample. The scatter in the absorption coefficient at these different positions was less than 5\%.

The nonlinear index of refraction was measured at $540 \mathrm{~nm}$ for the samples using the Z-scan method described previously. The laser used for these experiments was a cavity dumped tunable dye laser with a $\sim 6 \mathrm{ps}$ pulse duration. The laser was operated at $3.8 \mathrm{MHz}$. The average power 


\section{DISCLAMMER}

Portions of this document may be illegible in electronic image products. Images are produced from the best available original document. 
patterns from the samples reveal that two distinct metallic crystal structures exist in the implanted layer. The predominant features of the polycrystalline ring patterns were representative of the face centered cubic (FCC) Ag lattice and FCC metallic $\mathrm{Cu}$ lattice. The average particle size increases with increasing $\mathrm{Cu}$ dose. The mean size is $14.4,15.2$, and $17.5 \mathrm{~nm}$ respectively for samples with $\mathrm{Ag} / \mathrm{Cu}$ ratios of $9 / 3,6 / 6$, and $3 / 9$. The $9 / 3$ sample exhibits a bimodal distribution with the smallest distribution centered at $\sim 12 \mathrm{~nm}$ and the largest at $\sim 60 \mathrm{~nm}$. The $6 / 6 \mathrm{Ag} / \mathrm{Cu}$ sample exhibits a bimodal distribution with the smallest particles centered at a depth of $\sim 15 \mathrm{~nm}$ and the largest at $\sim 45$ $\mathrm{nm}$. The frequency of occurrence of particles in these two samples drops by a factor of $\sim 9$ for particle sizes in the part of the distribution containing the largest particles. The $3 / 9 \mathrm{ratio} \mathrm{Ag} / \mathrm{Cu}$ sample exhibits more of a skewed distribution centered at $\sim 17 \mathrm{~nm}$ than a bimodal distribution as observed in the other two samples. However, while small in number, the largest individual particles are in the sample with the $9 / 3$ ratio of $\mathrm{Ag} / \mathrm{Cu}$. The $6 / 6$ ratio sample has the next largest individual particles, and the $3 / 9$ sample the smallest individual particles.

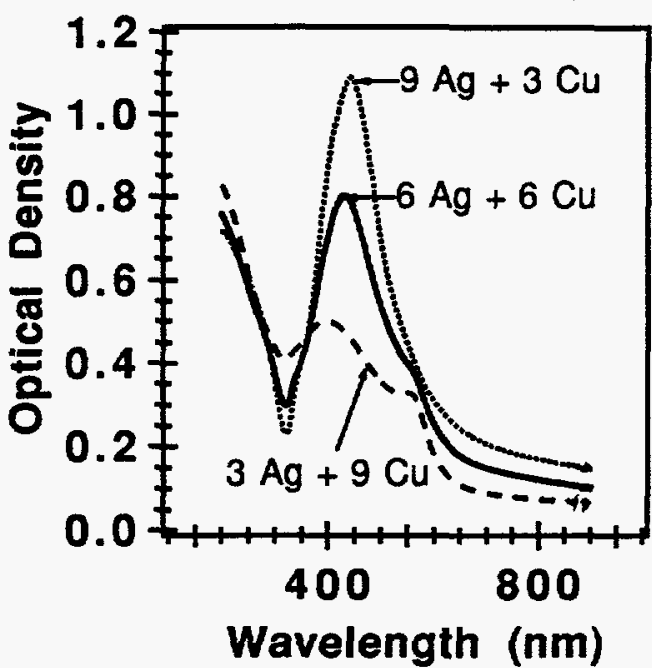

Figure 3. Optical Density as a function of wavelength for different ratios of $\mathrm{Ag}$ to $\mathrm{Cu}$.
The optical density for the three samples is shown in figure 3 as a function of wavelength. The dominant peak is at $-410 \mathrm{~nm}$ and shifts to longer wavelengths, $\sim 440 \mathrm{~nm}$ with increasing Ag concentration. A second peak at $\sim 575 \mathrm{~nm}$ grows with increasing $\mathrm{Cu}$ concentration. This peak appears as a shoulder in the lowest $\mathrm{Cu}$ dose sample, but is clearly discernible for the highest $\mathrm{Cu}$ dose. The values for the absorption coefficient, $\alpha$, at $570 \mathrm{~nm}$ are given in Table I. The normalized far field transmission for a small aperture as a function of sample position relative to the focal plane of the lens shows a decreasing then increasing intensity while moving through the focal plane indicating a positive intensity dependent index of refraction, $\mathrm{n}_{2}$. All samples displayed

similar behavior. The z-scan results can be related to $\mathrm{n}_{2}$ using the formalism of references. ${ }^{9,10}$ The values for $\mathrm{n}_{2}$ were calculated for a wavelength of $570 \mathrm{~nm}$ assuming an effective thickness of 0.15 microns. The values for $n_{2}$ increase with $\mathrm{Cu}$ dose and are given in Table $\mathrm{I}$.

Table I Linear and Nonlinear Optical Coefficients Measured at $570 \mathrm{~nm}$

\begin{tabular}{|l|l|c|c|}
\hline Sample & $\alpha\left(\mathrm{cm}^{-1}\right)$ & $\mathrm{n}_{2}\left(\mathrm{~cm}^{2} / \mathrm{W}\right)$ & $\beta(\mathrm{cm} / \mathrm{W})$ \\
\hline $9 \mathrm{Ag}$ to $3 \mathrm{Cu}$ & $5.9 \times 10^{4}$ & $1.0 \times 10^{-9}$ & $3.8 \times 10^{-5}$ \\
\hline $6 \mathrm{Ag}$ to $6 \mathrm{Cu}$ & $5.4 \times 10^{4}$ & $1.3 \times 10^{-9}$ & $-0.9 \times 10^{-5}$ \\
\hline $3 \mathrm{Ag}$ to $9 \mathrm{Cu}$ & $4.6 \times 10^{4}$ & $1.6 \times 10^{-9}$ & $-1.4 \times 10^{-5}$ \\
\hline
\end{tabular}

The samples were also measured using an open aperture. The open aperture measurements saturate for $\mathrm{Ag}$ doses $>6 \times 10^{16} \mathrm{Ag}$ ions $/ \mathrm{cm}^{2}$. From the open aperture results the two photon absorption coefficient, $\beta$, can be determined. Table I gives the values calculated for $\beta$ at $570 \mathrm{~nm}$.

\section{DISCUSSION}

Effective medium theory can be used to describe the optical response of nanometer dimension metal particles embedded in a dielectric medium. The linear response for colloids with diameters 
less than $\lambda / 20$, where $\lambda$ is the wavelength of the incident radiation, is reasonably described by Mie scattering theory in the electric dipole approximation, 11,12 and is given by

$$
\alpha=\frac{18 \pi n_{d}^{3}}{\lambda} \cdot \frac{p \varepsilon_{2}}{\left[\varepsilon_{1}+2 n_{d}^{2}\right]^{2}+\varepsilon_{2}^{2}}
$$

where $\alpha$ is the absorption coefficient, $\varepsilon(\lambda)=\varepsilon_{1}+i \varepsilon_{2}$ is the dielectric constant of the metal, $p$ is the volume fraction of the metal particles and $n_{d}$ is the index of refraction of the dielectric host. The absorption is expected to exhibit a peak at the surface plasmon resonance frequency for which the condition $\varepsilon_{1}+2 n_{\mathrm{d}}^{2}=0$ is met. The surface plasmon resonance frequency depends explicitly on the electronic properties of the metal colloids and on the index of refraction of the host dielectric, $n_{d}$, while depending implicitly on particle size.

The third order nonlinear susceptibility, $\chi_{\mathrm{eff}}^{(3)}$, of small non interacting particles in a dielectric can also be expressed using effective medium theory as 13

$$
\chi_{\text {eff }}^{(3)}=p f_{c}^{2}(\omega)\left|f_{2}(\omega)\right|^{2} \chi_{m}^{(3)} \text { and } f_{c}(\omega)=\frac{3 n_{d}^{2}}{\varepsilon_{1}+2 n_{d}^{2}}
$$

where $f_{c}(\omega)$ is the local field factor and $\chi_{m}^{(3)}$ is the nonlinear susceptibility of the metal clusters. There is a potentially large enhancement of the effective nonlinear susceptibility due to local field effects at surface plasmon resonance frequency. The index of refraction and the intensity dependent term are related to the above quantities by ${ }^{14}$

$$
\mathrm{n}=\mathrm{n}_{\mathrm{o}}+\mathrm{n}_{2} \mathrm{I} \quad \text { and } \quad \mathrm{n}_{2}=\frac{12 \pi}{\mathrm{n}_{\mathrm{o}}} \operatorname{Re}\left[\chi_{\text {eff }}^{(3)}\right]
$$

where $n_{0}$ is the linear index of refraction and $n_{2}$ is the intensity dependent component.

Previously we explained the optical absorption of sequentially implanted Ag and $\mathrm{Cu}$ samples with the same nominal dose by the formation of small alloy particles of $\mathrm{Ag}-\mathrm{Cu}<30 \mathrm{~nm}$ in mean diameter. We reported a shift in the surface plasmon resonance compared to samples implanted separately with $\mathrm{Ag}$ or $\mathrm{Cu}$ ions. This shift was attributed to the formation of a metastable $\mathrm{Ag}-\mathrm{Cu}$ alloy when the ions were sequentially implanted. Here we observe a small increasing blue shift and decreasing intensity with increasing $\mathrm{Cu}$ concentration for the peak at $\sim 440 \mathrm{~nm}$.. For the sample implanted with the highest dose of $\mathrm{Cu}$ ions a second peak is clearly discernible. A simple superposition of the absorption in samples with single element implantations at the same doses of $\mathrm{Ag}$ and $\mathrm{Cu}$ under the same conditions does not yield the absorption observed in the sequentially implanted samples. While the peak at $\sim 440 \mathrm{~nm}$ is similar to that due to $\mathrm{Ag}$ particles formed by single element Ag implantation into silica, the shifts to longer wavelengths and the changes in intensity and shape of the peak suggest that the $\mathrm{Ag}$ and $\mathrm{Cu}$ interact with each other. The peak at $-575 \mathrm{~nm}$ is similar to that in $\mathrm{Cu}$ implanted samples for similar doses, but is clearly affected by the presence of the $\mathrm{Ag}$. While some of the broad absorption exhibited by the $9 / 3 \mathrm{Ag} / \mathrm{Cu}$ sample on the long wavelength side of the spectrum may be due to the small number of larger particles formed, all the absorption spectra appear to be dominated by the more numerous small particles whose sizes are more nearly represented by the mean particle size. ${ }^{6}$ We conclude, based on the small differences in average particle size and the similar frequency of occurrence of the dominant particle sizes in all samples, that the changes observed in the linear optical spectra are due for the most part to interaction of the sequentially implanted ions to form metastable phases of varying concentrations of $\mathrm{Ag}$ and $\mathrm{Cu}$. The compositions formed depend on the relative amounts of $\mathrm{Ag}$ and Cu sequentially implanted.

The values calculated for $\mathrm{n}_{2}$ increase with increasing $\mathrm{Cu}$ dose and decreasing optical absorption as seen in Table I. Enhancements of the nonlinear susceptibility of the composite are expected on or near the surface plasmon resonance of the metal colloids. The measurements of $\mathrm{n}_{2}$ at $570 \mathrm{~nm}$ are 
closer to the surface plasmon resonance for $\mathrm{Cu}$ particles than they are to the surface plasmon resonance of $\mathrm{Ag}$ particles $(-400 \mathrm{~nm})$. We suggest that this increase in $\mathrm{n}_{2}$ is due to the presence of increasingly $\mathrm{Cu}$ rich particles with increasing $\mathrm{Cu}$ dose. However the presence of the $\mathrm{Ag}$ has a substantial impact on the nonlinear response of these composites. For a sample implanted with $\mathrm{Cu}$ to a dose of $6 \times 10^{16}$ ions $/ \mathrm{cm}^{2}$ under similar conditions, we obtain a value of $3.4 \times 10^{-10} \mathrm{~cm}^{2} / \mathrm{W}$ for $\mathrm{n}_{2}$. This is a factor of $\sim 5$ smaller than the sequentially implanted sample with the same $\mathrm{Cu}$ dose. While there is twice the nominal dose of metal ions present in the sequentially implanted sample, this factor of $\sim 5$ difference is too large to be accounted for by concentration differences alone, as $n_{2}$ increases linearly with volume fraction, $p$ (equation 1 ). We suggest the increase in $n_{2}$ is due to changes in the composition of the metal colloids with changing relative concentrations of implanted $\mathrm{Ag}$ to $\mathrm{Cu}$. The presence of the $\mathrm{Ag}$ ions appears to enhance the nonlinear response of the Cu near its surface plasmon resonance frequency.

The values for $n_{2}$ increase by $\sim 60 \%$ while the absorptivity decreases by $\sim 25 \%$ for the change in relative concentrations of $\mathrm{Ag}$ to $\mathrm{Cu}$ used in this work. We would expect the opposite behavior for an $n_{2}$ dominated by a thermal mechanisms, i.e., the values for $n_{2}$ would increase with increasing absorptivity due to increased thermal loading of the sample. As the electronic structure of the colloids and hence the optical response will be determined by the metal species, ${ }^{7,15}$ the observation that $\mathrm{n}_{2}$ increases with $\mathrm{Cu}$ to $\mathrm{Ag}$ ratio while $\alpha$ decreases indicates that the nonlinear response is related to the electronic structure of the colloids. Nonlinear measurements on $\mathrm{Cu}$ colloids formed by ion implantation have been reported previously. ${ }^{6}$ Using similar wavelength, power, rep rate, and pulse width of the laser, the thermal loading of the sample was found not to dominate the response because of the relatively long interpulse spacing ( $263 \mathrm{~ns}$ ), even though the 6 ps pulse duration is comparable to the electron thermalization times of the $\mathrm{Cu}$ colloids. Based on the above discussion we conclude that the nonlinear response observed for these sequentially implanted samples is dominated by an electronic mechanism. With increasing $\mathrm{Ag}$ dose $\beta$ saturates indicating a change in the nature of the two photon response. The role of the relative ratio of $\mathrm{Ag}$ to $\mathrm{Cu}$ in the two photon absorption processes is currently being studied.

In summary the presence of the Ag has a significant impact on the linear and nonlinear optical properties. These results suggest that it may be possible to significantly alter and enhance the optical responses of one metal colloid near its surface plasmon resonance frequency by the addition of a second element.

\section{ACKNOWLEDGMENTS}

The authors acknowledge the support of the Army Research Office under grant DAAH04-93-G0123 and Division of Materials Science, U.S. Department of Energy under contract DE-AC0584OR21400 with Martin-Marietta Energy Systems, Inc. We thank Prof. Richard Haglund for the use of his laboratory for the nonlinear measurements.

\section{REFERENCES}

1. R. H. Magruder,III, J.E. Wittig and R.A. Zuhr, J. Non Cryst. Solids, 163, 162 (1993).

2 .R.A. Zuhr, R.H. Magruder,III and J.E. Wittig, Mat. Res. Soc. Sym. Proc., 316, 457 (1994).

3. R.H. Magruder,III, R.A. Zuhr and D. O. Osborne submitted to Nucl. Instr. and Meth. B.

4. R.H. Magruder,III, R.F. Haglund, L. Yang, C.W. White, Lina Yang, R. Dorsinville and R.R. Alfano. J.Appl. Phys. Lett. 62, 465 (1993).

5. R.H. Magruder,III, D.L. Kinser, J.E. Wittig and R.A. Zuhr, SPIE Proceedings: Properties and Characteristics of Glass III, 1761180 (1992).

6. R.H. Magruder,II, R. F. Haglund, L.Yang,J.E. Wittig and R.A. Zuhr, J. Appl. Phys., 76, 715 (1994).

7. R.H. Magruder,III, D.O. Osborne and R.A. Zuhr, J. Non Cryst. Solids, in press.

8. J.P. Biersack and L.G. Haggmark, Nucl. Inst. Methods B 174, 257 (1980). 
9. M. Sheik-Bahae, A.A. Said, T. Wei, D.J. Hagan and E.W. Van Stryland, IEEE J. Quantum Electronics, 26, 760 (1990).

10. E.W. Van Stryland, M. Sheik-Bahae, A.A. Said, T. Wei, and D.J. Hagan, SPIE 1852, 135 (1993).

11. C.F. Bohren and D.R. Huffman, Absorption and Scattering of Light by Small Particles, (John Wiley and Sons, New York, 1983).

12. U. Kreibig and L. Genzel, Surf. Sci. 156, 678 (1985) .

13. F. Hache, D. Ricard, and C. Flytzanis, J. Opt. Soc. Am. B3, 1647 (1986).

14. M.J. Weber, D. Milam and W.L. Smith, Optical Eng., 17, 463 (1978).

15. R. E. Hummel, Electronic Properties of Materials, 2nd Ed., (Springer - Verlag, Berlin 1993).

\section{DISCLAIMER}

This report was prepared as an account of work sponsored by an agency of the United States Government. Neither the United States Government nor any agency thereof, nor any of their employees, makes any warranty, express or implied, or assumes any legal liability or responsibility for the accuracy, completeness, or usefulness of any information, apparatus, product, or process disclosed, or represents that its use would not infringe privately owned rights. Reference herein to any specific commercial product, process, or service by trade name, trademark, manufacturer, or otherwise does not necessarily constitute or imply its endorsement, recommendation, or favoring by the United States Government or any agency thereof. The views and opinions of authors expressed herein do not necessarily state or reflect those of the United States Government or any agency thereof. 